\title{
Participant List
}

Abraham Achterberg, Astronomical Institute, Utrecht University, Postbus 80.000, Utrecht, 3508 TA, Netherlands 〈a.achterberg@astro.uu.nl〉

Loren Acton, Montana State University, Department of Physics, Bozeman, MT 59717, U.S.A. 〈acton@physics.montana.edu〉

Steven Allen, Institute of Astronomy, Madingley Road, Cambridge, Cambridgeshire CB3 0HA, U.K. 〈swa@ast.cam.ac.uk〉

Abdalla AlMohd, Higher Institute of Astronomy and Space Sciences, PO Box 2275 , Irbid, Jordan 〈aseel@rocketmail.com〉

Elena Amato, University of Florence, Astronomy Department, Largo E. Fermi 5, Florence I-50125, Italy 〈amato@arcetri.astro.it〉

Seiichiro Aoki, The University of Tokyo, 2-17-10 Nishiasakusa, Taito-ku, Tokyo 111-0035, Japan 〈aoki@corona.mtk.nao.ac.jp〉

Keith Arnaud, Goddard Space Flight Center, Code 662, Greenbelt, MD 20771, U.S.A. 〈kaa@lheamail.gsfc.nasa.gov〉

Markus Aschwanden, Lockheed Martin Solar and Astrophysics Lab, 3251 Hanover St., Palo Alto, CA 94304. U.S.A. 〈aschwanden@lmsal.com〉

Marc Audard, Paul Scherrer Institute, ETH Zentrum, Zurich 8092, Switzerland 〈audard@astro.phys.ethz.ch〉

Rino Bandiera, Osservatorio Astrofisico di Arcetri, Largo E. Fermi 5, Firenze 50125, Italy 〈bandiera@arcetri.astro.it)

Lee Bargatze, Montana State University-Bozeman, 1302 Wildflower Way, Bozeman, MT 59715, U.S.A. 〈bargatze@mithra.physics.montana.edu〉

Manuel Bautista, NASA/GSFC, Code 662, Greenbelt, MD 20771, U.S.A. 〈bautista@lhea1.gsfc.nasa.gov 〉

Werner Becker, MPE-Garching, Giessenbachstrasse 1, Postfach 1603, Garching, Bavaria 85740, Germany〈web@mpe.mpg.de〉

John Belcher, Massachusetts Institute of Technology, Building 37, Room 695, Cambridge, MA 02139, U.S.A.〈jwb@space.mit.edu〉

Paul Bellaire, Air Force Office of Scientific Research, 801 N. Randolph St. Rm. 732, Arlington, VA 22203-1977, U.S.A. 〈paul.bellaire@afosr.af.mil〉

Jitesh Bhatt, Physical Research Laboratory, Navarangpura, Ahmedabad, Gujarat 380 009, India 〈jeet@prl.ernet.in〉

Eric Blackman, Cal Tech, Mail Code 130-33, Pasadena, CA 91125, U.S.A. 〈blackman@tapir.caltech.edu〉

Pasquale Blasi, University of Chicago, 5640 Ellis Ave., Chicago, IL 60637, U.S.A. 〈blasi@oddjob.uchicago.edu 〉

William Brandt, Penn State Astronomy, 525 Davey Lab, University Park, PA 16802, U.S.A. 〈niel@astro.psu〉

Wolfgang Brinkmann, MPI fuer extraterrestrische Physik, Postfach 1603, Garching, Bavaria D-85740, Germany 〈wpb@rzg.mpg.de〉 
Andrew Burnette, Montana State University, Department of Physics, Bozeman, MT 59717, U.S.A. 〈andy@mithra.physics.montana.edu〉

David Caditz, Montana State University, Department of Physics, Bozeman, MT 59717, U.S.A. 〈caditz@physics.montana.edu〉

Richard Canfield, Montana State University, Department of Physics, Bozeman, MT 59717, U.S.A. 〈canfield@physics.montana.edu 〉

Claude Canizares, Massachusetts Institute of Technology, 70 Vassar Street, Cambridge, MA 02139, U.S.A. 〈crc@space.mit.edu〉

K. S. Cheng, University of Hong Kong, Pokfulam Road, Hong Kong, China 〈hrspksc@hkucc.hku.hk〉

Roger Chevalier, University of Virginia, PO Box 3818, Charlottesville, VA 22903, U.S.A. 〈rac5x@virginia.edu〉

Monica Colpi, University of Milan, Department of Physics, Via Celoria 16, Milan 20133, Italy

Stirling Colgate, Los Alamos National Lab, MS B 288, LANL, Los Alamos, NM 87545, U.S.A. 〈colgate@lanl.gov〉

Antonia daCosta, Institute Superior Tecnico, Complexo I-CEIST, Lisboa 1049-001, Portugal 〈antonio_da_costa@ieee.org)

Alisdair Davey, Montana State University, Department of Physics, Bozeman, MT 59717, U.S.A. 〈ard@solar.physics.montana.edu〉

Tiziana DiMatteo, Harvard University, 60 Garden Street, Cambridge, MA 02138, U.S.A. 〈tdimatteo@cfa.harvard.edu〉

Taotao Fang, MIT, 37-624D, 70 Vassar Street, Cambridge, MA 02139, U.S.A. $\langle$ fangt@mit.edu

George Field, Center for Astrophysics, 60 Garden Street, Cambridge, MA 02138, U.S.A. 〈gfield@cfa.harvard.edu〉

Lucia Franco, University of Chicago, 5640 S. Ellis Ave., Chicago, IL 60637, U.S.A. 〈lucia@oddjob.uchicago.edu〉

Chris Fryer, UC Santa Cruz, UCO/Lick Observatory UCSC, Santa Cruz, CA 95064, U.S.A.〈cfryer@ucolick.org 〉

Keigo Fukumura, Montana State University, Department of Physics, Bozeman, MT 59717, U.S.A. 〈fukumura@physics.montana.edu 〉

Marek Gierlinski, Jagiellonian University Observatory, Orla 171, Krakow 30-244, Poland 〈gier@camk.edu.pl〉

Andrei Gruzinov, Institute for Advanced Study, Olden Lane, Princeton, NJ 08540, U.S.A. 〈andrei@ias.edu〉

Manuel Güdel, Paul Scherrer Institute, Wuerenlingen \& Villigen, Villigen PSI, AG 5232, Switzerland 〈guedel@astro.phys.ethz.ch〉

Daniel Harris, Smithsonian Astrophysical Observatory, MS-3 60 Garden St., Cambridge, MA 02138, U.S.A.

Kampalayya Hiremath, Indian Institute of Astrophysics, Bangalore, Karnataka 560034, India 〈 hiremath@iiap.ernet.in〉 
Kouichi Hirotani, National Astronomical Observatory, Osawa 2-21-1, Mitaka, Tokyo 181-8588, Japan 〈hirotani@hotaka.mtk.nao.ac.jp 〉

Stephen Holt, NASA/GSFC, Code 600, Greenbelt, MD 20904, U.S.A. 〈steve.holt@gsfc.nasa.gov〉

Yakov Istomin, Lebedev Physical Institute, Leninski Prosp, 53, Moscow, Moscow region 117924, Russia 〈istomin@td.lpi.ac.ru〉

Paul Joss, MIT, 77 Massachusetts Ave., Cambridge, MA 02139, U.S.A. 〈joss@mitlns.mit.edu 〉

Seiichi Kato, University of Tokyo, Hongo 7-3-1, Bunkyo-ku, Tokyo 113-0033, Japan 〈katosi@solar.mtk.nao.ac.jp〉

Vinod Krishan, Indian Institute of Astrophysics, Bangalore, Karnataka 560034, India〈vinod@iiap.ernet.in〉

Volodymyr Kryvdyk, Department of Astronomy, Kiev University, Glushkova 6, UKR-252022, Kiev, Ukraine〈kryvdyk@galaxy.ups.kiev.ua〉

Takahiro Kudoh, National Astronomical Observatory, Osawa 2-21-1, Mitka, Tokyo 181-8588, Japan 〈kudo@solar.mtk.nao.ac.jp )

Hideyo Kunieda, Nagoya University, Department of Astrophysics, Nagoya, Aichi 464-8602, Japan 〈kunieda@u.phys.nagoya-u.ac.jp〉

Erik Kuulkers, SRON, Sorbnnelaan 2, Utrecht 3584 CA, Netherlands $\langle$ e.kuulkers@sron.nl

Kenneth Lang, Tufts University, Dept. of Physics, Medford, MA 02155, U.S.A. 〈klang@emerald.tufts.edu 〉

Ted LaRosa, Kennesaw State University, 1000 Chastain Rd., Kennesaw, GA 30144, U.S.A. 〈ted@avatar.kennesaw.edu〉

Michelle Larson, Montana State University, Department of Physics, Bozeman, MT 59717, U.S.A. 〈mlarson@physics.montana.edu〉

Karen Leighly, Columbia University, 550 W. 120th St., New York, NY 10027, U.S.A. 〈leighly@ulisse.phys.columbia.edu 〉

Anna LeRoux, Montana State University, 19733 Spruce St., Castro Valley, CA 95456, U.S.A. 〈leroux@physics.montana.edu〉

Manfred Leubner, University of Innsbruck, Technikerstr. 25, Innsbruck, Tirol A-6020, Austria 〈manfred.leubner@uibk.ac.at〉

Hui Li, Los Alamos National Laboratory, T-6, MS B288, Los Alamos, NM 87545, U.S.A.〈hli@lanl.gov〉

Robert Lin, University of California, Space Sciences Lab, Berkeley, CA 94720-7450, U.S.A. 〈rlin@ssl.berkeley.edu 〉

Bennett Link, Montana State University, Department of Physics, Bozeman, MT 59717, U.S.A. 〈blink@dante.physics.montana.edu〉

Yuri Litvinenko, University of New Hampshire, UNH space Center, Durham, NH 03824-3525, U.S.A. 〈yuri.litvinenko@unh.edu 〉

Dana Longcope, Montana State University, Department of Physics, Bozeman, MT 59717, U.S.A. 〈dana@physics.montana.edu〉 
Maxim Lyutikov, Canadian Institute for Theoretical Astrophysics, 60 St. George Street, Toronto, Ontario M5S 3H8, Canada

〈lyutikov@cita.utoronto.ca〉

Feng Ma, University of Texas at Austin, Astronomy Department, Austin, TX 78712, U.S.A.〈feng@astro.as.utexas.edu〉

Yuqian Ma, Institute of High Energy Physics, PO Box 918-3, Beijing 100039, China 〈mayq@astrosv1.ihep.ac.cn〉

Andrew MacFadyen, UC Santa Cruz, Astronomy Department, Santa Cruz, CA 95064, U.S.A. 〈andrew@ucolick.org〉

Petrus Martens, Montana State University, Physics Department, Bozeman, MT 59717, U.S.A. 〈martens@physics.montana.edu〉

Satoshi Masuda, STEL, Nagoya University, Honohara 3-13, Toyokawa, Aichi 442-8507, Japan 〈masuda@stelab.nagoya-u.ac.)

David McKenzie, Montana State University, Physics Department, Bozeman, MT 59717, U.S.A. 〈mckenzie@physics.montana.edu〉

Ivy Merriot, 1302 Wildflower Way, Bozeman, MT 59715, U.S.A.

Robyn Millan, U.C. Berkeley, Space Sciences Lab, Berkeley, CA 94720-7450, U.S.A. 〈rmillan@ssl.berkeley.edu〉

James Miller, University of Alabama in Huntsville, Department of Physics, Huntsville, AL 35899, U.S.A. 〈miller@mpingo.uah.edu〉

Raquel Morales, Institute of Astronomy, Madingley Rd., Cambridge, Cambridgeshire CB3 0HA, U.K. 〈rm@ast.cam.ac.uk〉

Takayoski Nakamura, University of Tokyo, Department of Astronomy, Bunkyo Ward, Tokyo 113-0033, Japan 〈nakamura@astron.s.u-tokyo.ac.jp 〉

Shin-ya Nitta, National Astronomical Observatory, Division of Theoretical Astrophysics, Mitaka, Tokyo 181-8588, Japan〈snitta@th.nao.ac.jp )

Elizabeth Noonan, Montana State University, Physics Dept., PO Box 173840, Bozeman, MT 59717, U.S.A.〈ejnoonan@solar.physics.montana.edu〉

Jay Norris, NASA/Goddard Space Flight Center, Code 661, Greenbelt, MD 20771, U.S.A.〈norris@groax0.gsfc.nasa.gov〉

Angela Olinto, University of Chicago, 5640 S. Ellis Ave., AAC-142, Chicago, IL 60605, U.S.A. 〈olinto@oddjob.uchicago.edu〉

Erella Opher, University of Sao Paulo, Av. Miguel Stefano 4200, Sao Paulo, SP 04301, Brazil〈erella@orion.iagusp.usp.br〉

Reuven Opher, University of Sao Paulo, Av. Miguel Stefano 4200, Sao Paulo, SP 04301, Brazil 〈opher@orion.iagusp.usp.br〉

Merav Opher, University of Sao Paulo, Av. Higienopolis 794, Sao Paulo, SP 01238, Brazil〈merav@orion.iagusp.usp.br〉

Franco Pacini, Arcetri Astrophysical Observatory, Largo Fermi 5, Firenze, Tuscany 50125, Italy 〈pacini@arcetri.astro.it〉

Eugene Parker, University of Chicago, 1323 Evergreen Rd., Homewood, IL 60430, U.S.A. 〈parker@odysseus.uchicago.edu〉 
George Pavlov, Pennsylvania State University, 525 Davey Lab, University Park, PA 16802, U.S.A. 〈pavlov@astro.psu.edu〉

Qiu-he Peng, Nanjing University, Department of Astronomy, Nanjing 210093, China 〈qhpeng@nju.edu.cn〉

Robert Petre, NASA/GSFC, Code 662, Greenbelt, MD 20771, U.S.A. 〈rob@hatrack.gsfc.nasa.gov〉

Alexei Pevtsov, Montana State University, Physics Dept., PO Box 173840, Bozeman, MT 59717, U.S.A. 〈pevtsov@physics.montana.edu〉

Luigi Piro, Istituto Astrofisica Spaziale, CNR Rome, Via Fosso del Cavaliere, Roma RM I-00133, Italy〈piro@ias.rm.cnr.it〉

Sergei Popov, Sternberg Astronomical Institute, Universitetskii pr 13, Moscow 119899, Russia 〈polar@sai.msu.su〉

Dfimitrios Psaltis, Harvard-Smithsonian CFA, 60 Garden St., MS-51, Cambridge, MA 02138, U.S.A. 〈dpsaltis@cfa.harvard.edu〉

Reuven Ramaty, NASA/GSFC, Code 661, Greenbelt, MD 20771, U.S.A. 〈ramaty@gsfc.nasa.gov〉

Thomas Rauch, Inst. F. Astronomie u. Astrophysik d. Universitaet, Waldhaeuser Strasse 64, Tuebingen D-72411, Germany 〈rauch@astro.uni-tuebingen.de〉

Alak Ray, Tata Institute of Fundamental Research, Homi Bhabaha Rd., Bombay, Maharashtra 400 005, India〈akr@dashoo.tifr.res.in〉

Darrell Rilett, Montana State University, Physics Dept., PO Box 173840, Bozeman, MT 59717, U.S.A. 〈rilett@physics.montana.edu〉

Roger Romani, Stanford University, Department of Physics, MC 4060, Stanford, CA 94305-4060, U.S.A. 〈rwr@astro.stanford.edu〉

Robert Rosner, University of Chicago, 5640 S. Ellis Ave., Chicago, IL 60637, U.S.A. 〈r-rosner@uchicago.edu〉

Malvin Ruderman, Columbia University, Department of Physics, New York, NY 10027, U.S.A. 〈mar@astro.columbia.edu〉

Ravi Sankrit, The Johns Hopkins University, 3400 N. Charles St., Baltimore, MD 21218, U.S.A. 〈ravi@pha.jhu.edu〉

Re'em Sari, Cal Tech, Theoretical Astrophysics 130-33, 1200 E. California Blvd., Pasadena, CA 91125, U.S.A. 〈sari@tapir.caltech.edu)

Kazunari Shibata, Kwasan and Hida Observatories, Kyoto University, Yamashina, Kyota 607-8471, Japan

Steven Shore, Indiana University, 1700 Mishawaka Ave., South Bend, IN 46634-7111, U.S.A. 〈sshore@paladin.iusb.edu〉

Ran Sivron, Bucknell University, Physics Department, Lewisburg, PA 17837, U.S.A.〈rsivron@bucknell.edu〉

Ravi Sood, Australian Defense Force Academy, School of Physics, Northcott Dr., Canberra, ACT 2600, Australia〈r.sood@adfa.edu.au〉 
Hendrik Spruit, MPI f. Astrophysik, Postfach 1523, Garching bei Muenchen D-85740, Germany 〈henk@mpa-garching.mpg.de〉

Gangsan Srinivasan, Rama Research Institute, C.V. Raman Avenue, Sadahivanagar, Bangalorg, Karnataka 5600801, India 〈srini@rri.ernet.in〉

Michael Stage, Center for Space Research, 77 Massachusetts Ave., Cambridge, MA 02139, U.S.A. 〈mikstage@mit.edu〉

Firoza Sutaria, IUCAA, Post Bag No. 4, Pune, Maharashtra 411-007, India $\langle$ fks@iucaa.ernet.in $\rangle$

Roland Svensson, Stockholm Observatory, Saltsjobaden SE 133 36, Sweden 〈svensson@astro.su.se〉

Jean Swank, NASA's GSFC/LHEA, Code 662, Greenbelt, MD 20771, U.S.A. 〈swank@pcasun1.gsfc.nasa.gov〉

Masaaki Takahashi, Aichi University of Education, Aichi University, Kariya, Aichi 448-8542, Japan 〈mtakahas@auecc.aichi-edu.ac.jp 〉

Yasuo Tanaka, Max Planck Institut fuer Extraterretrische Physik, Giessenbachstrasse, Garching D-85748, Germany

〈ytanaka@xray.mpe.mpg.de〉

Marcus Teter, Montana State University, Department of Physics, Bozeman, MT 59717, U.S.A. 〈teter@agn.physics.montana.edu〉

Christopher Thompson, University of North Carolina, Physics and Astronomy Department, Chapel Hill, NC 27599, U.S.A. 〈act@physics.unc.edu〉

Ulf Torkelsson, Chalmers University of Technology, Department of Theoretical Physics, Gothenburg 41296, Sweden〈torkel@fy.chalmers.se〉

Sachiko Tsuruta, Montana State University, Department of Physics, Bozeman, MT 59717, U.S.A. 〈tsuruta@physics.montana.edu 〉

Yutaka Uchida, Science University of Tokyo, 1-3, Kagurazaka, Shinjuku-ku, Tokyo 162, Japan〈es3y-ucd@asahi-net.or.jp〉

Frederick Walter, SUNY Stony Brook, Department of Physics, Stony Brook, NY 11794-3800, U.S.A. 〈fwalter@astro.sunysb.edu〉

De Yu Wang, Purple Mountain Observatory, 2, West Beijing Rd., Nanjing 210008, China 〈wangdeyu@jlonline.com)

Zhenru Wang, Nanjing University, Department of Astronomy, Nanjinig, Jiangsu 210093, China 〈zrwang@nju.edu.cn〉

Grzegorz Wardzinski, N. Copernicus Astronomical Center, CAMK, ul. Bartycka 18, Warszawa 00-716, Poland 〈gwar@camk.edu.pl〉

Brian Welsch, Montana State University, Department of Physics, Bozeman, MT 59717, U.S.A. 〈welsch@physics.montana.edu〉

Nicholas White, NASA/GSFC, Code 662, Greenbelt, MD 20771, U.S.A. 〈nwhite@lheapop.gsfc.nasa.gov >

Meredith Wills-Davey, Montana State University, Department of Physics, Bozeman, MT 59717, U.S.A. 〈meredith@solar.physics.montana.edu〉 
Takaaki Yokoyama, National Astronomical Observatory, Nobeyama Radio Astronomy, Minamimaki, Nagano 384-1305, Japan

〈yokoyama@solar.mtk.nao.ac.jp 〉

Andrzej Zdziarski, Copernicus Astronomical Center, Bartycka 18, Warsaw 00-716, Poland 〈aaz@camk.edu.pl〉 